\title{
High Contiguity de novo Genome Sequence Assembly of Trifoliate Yam (Dioscorea dumetorum) Using Long Read Sequencing
}

\author{
Christian Siadjeu ${ }^{1,2,+}$,, Boas Pucker ${ }^{2,3,+}{ }^{\circ}$, Prisca Viehöver ${ }^{2}$, Dirk C. Albach ${ }^{1}{ }^{(D}$ and \\ Bernd Weisshaar $2, *$ (D) \\ 1 Institute for Biology and Environmental Sciences, Biodiversity and Evolution of Plants, Carl-von-Ossietzky \\ University Oldenburg, Carl-von-Ossietzky Str. 9-11, 26111 Oldenburg, Germany; \\ christian.siadjeu@uol.de (C.S.); dirk.albach@uol.de (D.C.A.) \\ 2 Genetics and Genomics of Plants, Faculty of Biology, Center for Biotechnology (CeBiTec), \\ Bielefeld University, Sequenz 1, 33615 Bielefeld, NRW, Germany; bpucker@cebitec.uni-bielefeld.de (B.P.); \\ viehoeve@cebitec.uni-bielefeld.de (P.V.) \\ 3 Molecular Genetics and Physiology of Plants, Faculty of Biology and Biotechnology, \\ Ruhr-University Bochum, Universitätsstraße 150, 44801 Bochum, Germany \\ * Correspondence: bernd.weisshaar@uni-bielefeld.de; Tel.: +49-521-106-8720 \\ + These authors contributed equally to this work.
}

Received: 31 January 2020; Accepted: 29 February 2020; Published: 4 March 2020

\begin{abstract}
Trifoliate yam (Dioscorea dumetorum) is one example of an orphan crop, not traded internationally. Post-harvest hardening of the tubers of this species starts within $24 \mathrm{~h}$ after harvesting and renders the tubers inedible. Genomic resources are required for $D$. dumetorum to improve breeding for non-hardening varieties as well as for other traits. We sequenced the D. dumetorum genome and generated the corresponding annotation. The two haplophases of this highly heterozygous genome were separated to a large extent. The assembly represents $485 \mathrm{Mbp}$ of the genome with an $\mathrm{N}_{50}$ of over 3.2 Mbp. A total of 35,269 protein-encoding gene models as well as 9941 non-coding RNA genes were predicted, and functional annotations were assigned.
\end{abstract}

Keywords: yam; D. dumetorum; nanopore sequencing; genome assembly; comparative genomics; read depth

\section{Introduction}

The yam species Dioscorea dumetorum (Trifoliate yam) belongs to the genus Dioscorea comprising about 600 described species. The genus is widely distributed throughout the tropics [1] and includes important root crops that offer staple food for over 300 million people. Eight Dioscorea species are commonly consumed in West and Central Africa, of which D. dumetorum has the highest nutritional value [2]. Tubers of $D$. dumetorum are protein-rich (9.6\%) with a fairly balanced essential amino acids composition [3]. The provitamin A and carotenoid contents of the tubers of deep yellow genotypes are equivalent to those of yellow corn maize lines selected for increased concentrations of provitamin A [4]. The deep yellow yam tubers are used in antidiabetic treatments in Nigeria [5], probably due to the presence of dioscoretine, which is a bioactive compound with hypoglycaemic properties [6]. Yet, D. dumetorum constitutes an underutilized and neglected crop species despite its great potential for nutritional, agricultural, and pharmaceutical purposes.

Unlike other yam species, the agricultural value of $D$. dumetorum is limited by post-harvest hardening, which starts within $24 \mathrm{~h}$ after harvest and renders tubers inedible. Previous research showed that among $32 \mathrm{D}$. dumetorum cultivars tested, one cultivar was not affected by the hardening 
phenomenon [7]. This discovery provides a starting point for a breeding program of D. dumetorum against the post-harvest hardening phenomenon. Dioscorea cultivars are obligate outcrossing plants that display highly heterozygous genomes. Thus, methods of genetic analysis routinely applied in inbreeding species such as linkage analysis using the segregation patterns of an F2 generation or recombinant inbred lines are inapplicable to yam [8]. Furthermore, the development of marker-assisted selection requires the establishment of marker assays and dense genetic linkage maps. Thus, access to a complete and well-annotated genome sequence is one essential step towards the implementation of comprehensive genetic, genomic, and population genomic approaches for D. dumetorum breeding. So far, a genome sequence assembly for Dioscorea rotundata (Guinea yam) [8] and a reference genetic map for Dioscorea alata (Greater yam) [9] have been released. However, these two species belong to the same section of Dioscorea (D. sect. Enantiophyllum) but are distant from D. dumetorum (D. sect. Lasiophyton) in phylogenetic analyses $[10,11]$. They also differ in chromosome number $[8,12,13]$ making it unlikely that genetic maps can be directly transferred to $D$. dumetorum. Here, we report long read sequencing and de novo genome sequence assembly of the cultivar $D$. dumetorum Ibo sweet 3 that does not display post-harvest hardening.

\section{Materials and Methods}

\subsection{Sampling and Sequencing}

The D. dumetorum accession Ibo sweet 3 that does not display post-harvest hardening had been collected in the South-West region of Cameroon in 2013 [7]. Tubers of this accession were transferred to Oldenburg (Germany) and the corresponding plants were cultivated in a greenhouse at $25^{\circ} \mathrm{C}$. The haploid genome size of the Ibo sweet 3 genotype had been estimated to be $322 \mathrm{Mbp}$ through flow cytometry [14].

High molecular weight DNA was extracted from $1 \mathrm{~g}$ of leaf tissue using a CTAB-based method modified from [15]. After grinding the sample in liquid nitrogen, the powder was suspended in $5 \mathrm{~mL}$ CTAB1 (100 mM Tris-HCl pH 8.0, 20 mM EDTA, 1.4 M NaCl, 2\% CTAB, 0.25\% PVP) buffer supplemented with $300 \mu \mathrm{L} \beta$-mercaptoethanol. The suspension was incubated at $75^{\circ} \mathrm{C}$ for $30 \mathrm{~min}$ and inverted every $5 \mathrm{~min}$. Next, $5 \mathrm{~mL}$ dichloromethane was added and the solutions were gently mixed by inverting. The sample was centrifuged at $12,000 \mathrm{~g}$ at $20{ }^{\circ} \mathrm{C}$ for $30 \mathrm{~min}$. The clear supernatant was mixed with $10 \mathrm{~mL}$ CTAB2 (50 mM Tris- $\mathrm{HCl} \mathrm{pH}$ 8.0, $10 \mathrm{mM}$ EDTA, 1\% CTAB, $0.125 \% \mathrm{PVP}$ ) in a new reaction tube by inverting. Next, a centrifugation was performed at $12,000 \mathrm{~g}$ at $20^{\circ} \mathrm{C}$ for $30 \mathrm{~min}$. After discarding the supernatant, $1 \mathrm{~mL} \mathrm{NaCl}(1 \mathrm{M})$ was added to re-suspend the sediment by gently flicking the tube. By adding an equivalent amount of $1 \mathrm{~mL}$ isopropanol and careful mixing, the DNA was precipitated again and the sample was centrifuged as described above. After washing the sediment with $1 \mathrm{~mL}$ of $70 \%$ ethanol, $200 \mu \mathrm{L}$ TE buffer (10 mM Tris pH 8.0, $0.1 \mathrm{mM}$ EDTA) containing $2 \mathrm{mg}$ DNAse-free RNaseA were added. Re-suspension and RNA degradation were achieved by incubation overnight at room temperature. DNA quality and quantity were assessed via NanoDrop2000 (Thermo Fisher Scientific, Waltham, MA, USA) measurement, agarose gel electrophoresis, and Qubit (Invitrogen, Carlsbad, CA, USA) measurement. The short read eliminator (SRE) kit (Circulomics, Baltimore, MD, USA) was used to enrich long DNA fragments following the suppliers' instructions. Results were validated via Qubit measurement.

Library preparation was performed with $1 \mu \mathrm{g}$ of high molecular weight DNA following the SQK-LSK109 protocol (Oxford Nanopore Technologies, ONT, Oxford, UK). Sequencing was performed on four R9.4.1 flow cells on a GridION. Flow cells were treated with nuclease flush ( $20 \mu \mathrm{L}$ DNaseI (New England Biolabs (NEB), Ipswich, MA, USA) and $380 \mu \mathrm{L}$ nuclease flush buffer) once the number of active pores dropped below 200, to allow successive sequencing of multiple libraries on an individual flow cell. Live base calling was performed on the GridION by Guppy v3.0 (ONT).

A total of $200 \mathrm{ng}$ high molecular weight gDNA was fragmented by sonication using a Bioruptor (Diagenode) and subsequently used for Illumina library preparation. End-repaired fragments were 
size selected by AmpureXp Beads (Beckmann-Coulter) to an average size of $650 \mathrm{bp}$. After A-tailing and adaptor ligation fragments that carried adaptors on both ends were enriched by eight cycles of PCR (TruSeq Nano DNA Sample Kit; Illumina, San Diego, CA, USA). The final library was quantified using PicoGreen (Quant-iT, Invitrogen, CA, USA) on a FLUOstar plate reader (BMG labtech, Ortenberg, Germany) and quality checked by HS-Chips on a 2100 Bioanalyzer (Agilent Technologies, Santa Clara, CA, USA). The PE library was sequenced in $2 \times 250$ nt mode on an Illumina HiSeq-1500 instrument.

\subsection{Genome Assembly and Polishing}

Genome size prediction was performed with GenomeScope [16], findGSE [17], and gce [18] based on k-mer histograms generated by JellyFish v2 [19] as previously described [20] for different k-mer size values. In addition, MGSE [20] was run on an Illumina read mapping with single copy BUSCOs as reference regions for the haploid coverage calculation. Smudgeplot [21] was run on the same k-mer histograms (also for different k-mer size values) as the genome size estimations to estimate the ploidy.

Canu v1.8 [22] was deployed for the genome assembly. Raw ONT reads were provided as input to Canu for correction and trimming. Subsequently, Canu assembled the genome sequence from the resulting polished reads. The following optimized parameters were used "'genomeSize = $350 \mathrm{~m}$ ', 'corOutCoverage $=200$ ' 'correctedErrorRate $=0.12$ ' batOptions $=-\mathrm{dg} 3-\mathrm{db} 3-\mathrm{dr} 1-\mathrm{ca} 500-\mathrm{cp}$ 50 ' 'minReadLength $=10000$ ' 'minOverlapLength $=5000$ ' 'corMhapFilterThreshold $=0.0000000002$ ' 'ovlMerThreshold = 500' 'corMhapOptions = -threshold 0.85 -num-hashes 512 -num-min-matches 3 -ordered-sketch-size 1000 -ordered-kmer-size 14 -min-olap-length 5000 -repeat-idf-scale 50'”. The selected parameters were optimized for the assembly of a heterozygous genome sequence and our data set. The value for the genome size, estimated to be $322 \mathrm{Mbp}$, was increased to $350 \mathrm{Mbp}$ to increase the number of reads utilized for the assembly process. A total of $66.7 \mathrm{Gbp}$ of ONT reads with an $\mathrm{N}_{50}$ of $23 \mathrm{kbp}$ was used for assembly, correction, and trimming.

ONT reads were mapped back to the assembled sequence with minimap2 v2.17 [23], using the settings recommended for ONT reads. Next, the contigs were polished with racon v.1.4.7 [24] with $-\mathrm{m} 8-\mathrm{x}-6-\mathrm{g}-8$ as recommended prior to the polishing step with medaka. Two runs of medaka v.0.10.0 (https://github.com/nanoporetech/medaka) polishing were performed with default parameters (-m r941_min_high) using ONT reads. Illumina short reads were aligned to the medaka consensus sequence using BWA-MEM v. 0.7.17 [25]. This alignment was subjected to Pilon v1.23 [26] for final polishing in three iterative rounds with default parameters for the correction of all variant types and -mindepth 4 .

Downstream processing was based on a previously described workflow [27] and was performed by customized Python scripts for purging of contigs shorter than $100 \mathrm{kbp}$ and calculation of assembly statistics (https://github.com/bpucker/yam). In general, sequences were kept if matching a white list (D. rotundata) and discarded if matching a black list (bacterial/fungal genome sequences). Sequences with perfect matches against the genome sequences of plants that were sequenced in the lab in parallel (Arabidopsis thaliana, Beta vulgaris, and Vitis vinifera) were discarded as well. Contigs with less than 3-fold average coverage in an Illumina short read mapping were compared against nt via BLASTn with an e-value cut-off at $10^{-10}$ to identify and remove additional bacterial and fungal sequences.

For sorting ("scaffolding" according to linkage groups) the D. dumetorum assembly we employed D. rotundata pseudochromosomes. D. rotundata pseudochromosome sequences longer than $100 \mathrm{kbp}$ were split into chunks of $1000 \mathrm{bp}$ and subject to a BLASTn search against the D. dumetorum assembly with a word size of 12 . Hits were considered if the similarity was at least $70 \%$ and if at least $70 \%$ of the query length were covered by the alignment. To avoid ambiguous hits against close paralogs or between repeat units, BLAST hits were excluded if the second hit exceeded $90 \%$ of the score of the top hit. The known order of all chunks on the D. rotundata sequence was considered as a "pseudo genetic map" to arrange the D. dumetorum contigs via ALLMAPS v0.9.14 [28]. 


\subsection{Genome Sequence Annotation}

Hints for gene prediction were generated by aligning $D$. rotundata transcript sequences (TDr96 v1.0) [8] as previously described [29]. BUSCO v3 [30] was applied to generate a species-specific set of AUGUSTUS gene prediction parameter files. For comparison of annotation results, the D. rotundata genome assembly GCA_002260605.1 [8] was retrieved from NCBI. Gene prediction hints of D. dumetorum and dedicated parameters were subjected to AUGUSTUS v.3.3 [31] for gene prediction with previously described settings [29]. Various approaches involving AUGUSTUS parameter files for rice and maize genome sequences provided by AUGUSTUS, as well as running the gene prediction on a sequence with repeats masked by RepeatMasker v4.0.8 [32] with default parameters, were evaluated. BUSCO was applied repeatedly to assess the completeness of the gene predictions. The best results for D. dumetorum genome sequence annotation were obtained by using an unmasked assembly sequence and by applying yam specific AUGUSTUS gene prediction parameter files generated via BUSCO as previously described $[30,33]$. Predicted genes were filtered based on sequence similarity to entries in several databases (UniProt/SwissProt, Araport11, Brachypodium distachyon v3.0, Elaeis guineensis v5.1, GCF_000005425.2, GCF_000413155.1, Musa acuminata Pahang v2). Predicted peptide sequences were compared to these databases via BLASTp [34] using an e-value cut-off of $10^{-5}$. Scores of resulting BLASTp hits were normalized to the score when searched against the set of predicted peptides. Only predicted sequences with at least 0.25 score ratio and 0.25 query length covered by the best alignment were kept. Representative transcript and peptide sequences were identified per gene to encode the longest possible peptide as previously established [29,35]. GO terms were assigned via InterProScan5 [36]. Reciprocal best BLAST hits against Araport11 [35] were identified based on a previously developed script [27]. Remaining sequences were annotated via best BLAST hits against Araport11 with an e-value cut-off at 0.0001. The Araport11 annotation was transferred to predicted sequences.

Prediction of non-protein coding RNA genes like tRNA and rRNA genes was performed based on tRNAscan-SE v2.0.3 [37,38] and INFERNAL (cmscan) v1.1.2 [39] based on Rfam13 [40].

RepeatModeler v2 [41] was deployed with default settings for the identification of repeat family consensus sequences.

\subsection{Assembly and Annotation Assessment}

The percentage of phase-separated and merged regions in the genome assembly was assessed with the focus on predicted genes. Based on Illumina and ONT read mappings, the average coverage depth per gene was calculated. The distribution of these average values per gene allowed the classification of genes as phase-separated (haploid read depth) or merged (diploid read depth). As previous studies revealed that Illumina short reads have a higher resolution for such coverage analysis [42], we focused on the Illumina read data set for these analyses. Sequence variants were detected based on this read mapping as previously described [43]. The number of heterozygous variants per gene was calculated and compared between the groups of putatively phase-separated and merged genes. Predicted peptide sequences were compared against the annotation of other species including A. thaliana and D. rotundata via OrthoFinder v2 [44].

Sequence reads and assembled sequences are available at ENA under the project ID ERP118030 (see File S1 for details). The assembly described in this manuscript is available under GCA_902712375. Additional annotation files including the contigs assigned to organelle genomes are available as a data publication from the institutional repository of Bielefeld University at https://doi.org/10.4119/unibi/2941469.

Alleles covered by the fraction of phase-separated gene models were matched based on reciprocal best BLAST hits of the coding sequences (CDSs) following a previously described approach [27]. Alleles were considered a valid pair that represents a single gene if the second best match displayed $99 \%$ or less of the score of the best match. A customized Python script for this allele assignment is available on github (https://github.com/bpucker/yam). 


\section{Results}

In total, we generated $66.7 \mathrm{Gbp}$ of ONT reads data representing about 207× coverage of the estimated $322 \mathrm{Mbp}$ haploid D. dumetorum genome. Read length $\mathrm{N}_{50}$ of the raw ONT data set was $23 \mathrm{kbp}$ and increased to $38 \mathrm{kbp}$ through correction, trimming, and filtering. Additionally, $30 \mathrm{Gbp}$ of Illumina short read data (about $100 \times$ coverage) were generated. After polishing, the final assembly represents $485 \mathrm{Mbp}$ of the highly heterozygous D. dumetorum genome with an $\mathrm{N}_{50}$ of $3.2 \mathrm{Mbp}$ (Table 1 ). Substantial improvement of the initial assembly through various polishing steps was indicated by the increasing number of recovered BUSCOs (File S2). The final assembly displayed more BUSCOs ( $92.30 \%$ out of 1440 included in the embryophyta data set, see File S2 for details on the various BUSCO classes) compared to the publicly available genome sequence assembly of $D$. rotundata (v0.1) for that we detected $81.70 \%$ BUSCOs with identical parameters. Since there is no genetic map available for $D$. dumetorum, we transferred linkage group assignments from $D$. rotundata to our assembly. In total, 206 contigs comprising $330 \mathrm{Mbp}$ were assigned to a linkage group, while 718 contigs remained unplaced with a total sequence of $155 \mathrm{Mbp}$ (File S3). One plastid and six mitochondrial contigs were identified based on sequence similarity to $D$. rotundata organelle genome sequences (see https://doi.org/10.4119/unibi/2941469); the assignment was confirmed by very high coverage in the read mapping. Our D. dumetorum plastid sequence turned out to be almost identical to the data recently provided for the D. dumetorum plastome [11].

Table 1. Statistics of selected versions of the Dioscorea dumetorum genome assembly (see File S5 for a full table).

\begin{tabular}{cccccc}
\hline & Initial Assembly & Racon1 & Medaka2 & Pilon3 & Final \\
\hline Number of contigs & 1172 & 1172 & 1215 & 1215 & 924 \\
Max. contig length (bp) & $20,187,448$ & $20,424,333$ & $17,910,017$ & $17,878,854$ & $17,878,854$ \\
Assembly size (bp) & $501,985,705$ & $508,061,170$ & $507,215,754$ & $506,184,192$ & $485,115,345$ \\
Assembly size without N (bp) & $501,985,705$ & $508,061,170$ & $507,215,754$ & $506,184,192$ & $485,115,345$ \\
GC content & $37.74 \%$ & $37.66 \%$ & $37.87 \%$ & $37.59 \%$ & $37.57 \%$ \\
$N_{50}$ (bp) & $3,896,882$ & $3,930,287$ & $2,598,889$ & $2,593,751$ & $3,190,870$ \\
$N_{90}$ (bp) & 136,614 & 138,199 & 137,206 & 136,754 & 156,407 \\
BUSCO (complete) & $85.70 \%$ & $89.80 \%$ & $91.90 \%$ & $92.30 \%$ & $92.30 \%$ \\
\hline
\end{tabular}

Haploid genome size estimations based on k-mer distributions of the Illumina sequence reads ranged from $215 \mathrm{Mbp}$ (gce) over $254 \mathrm{Mbp}$ (GenomeScope) to $350 \mathrm{Mbp}$ (findGSE, MGSE) (File S4). The differences between the estimates might be influenced by the repeat content of the D. dumetorum genome (see below).

Different gene prediction approaches were evaluated (File S6) leading to a final set of 35,269 protein-encoding gene models. The average gene model spans $4.3 \mathrm{kbp}$, comprises six exons and encodes 455 amino acids (see File $\mathrm{S} 6$ for details). The gene prediction dataset for D. dumetorum is further supported by the identification of 6475 single copy orthologs between $D$. dumetorum and D. rotundata as well as additional orthogroups (File S7). Based on these single copy orthologs, the similarity of $D$. dumetorum and D. rotundata sequences was determined to be mostly above $80 \%$ (File S8). If the phase-separated allelic gene models were considered (Figure 1), 3352 additional single copy orthologs were detected. Functional annotation was assigned to 23,835 gene models (File S9). Additionally, 9941 non-coding RNA gene models were predicted including 784 putative tRNA genes (see https://doi.org/10.4119/unibi/2941469). Finally, and in addition to gene models encoding proteins and various RNA types, we identified 1129 repeat consensus sequences with a combined length of $1.3 \mathrm{Mbp}$ (File S10). The maximal repeat consensus length is $17.4 \mathrm{kbp}$, while the $\mathrm{N}_{50}$ is only $2.5 \mathrm{kbp}$.

Average read mapping depth per gene was analyzed to distinguish genes annotated in separated haplophases as well as merged sequences, respectively (Figure 1, File S11). About $64 \%$ of all predicted protein-encoding gene models were in the expected range of the haploid read mapping depth between 50 -fold and 150 -fold and about $27 \%$ are merged with a read depth between 150 -fold and 250 -fold. Only $6 \%$ of all genes show an average read depth below 50 -fold and only $1 \%$ show an average coverage 
higher than 250-fold. It should be noted that the gene models annotated in the phase separated part will cover in general two alleles per gene. A total of 22,885 gene models, representing the $64 \%$ in the range of the haploid read mapping depth, were sorted into allelic pairs which was successful for 8492 genes. The findings presented above can be explained by a diploid genome. An analysis with Smudgeplot indicated hints for a tetraploid genome from analysis with a k-mer size of 19, while the other three investigated k-mer sizes supported a diploid genome (File S12).

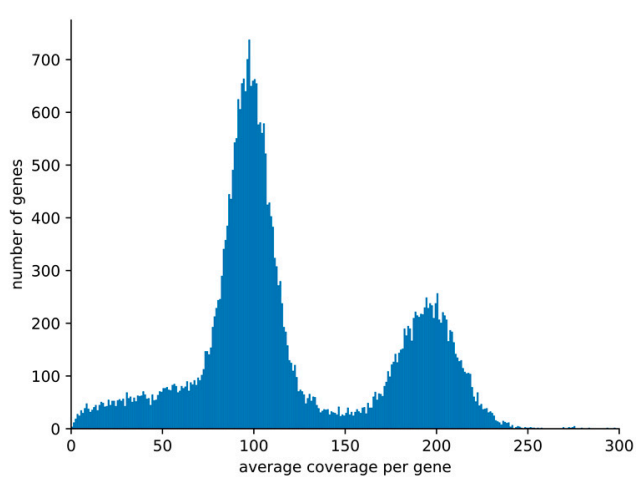

(a)

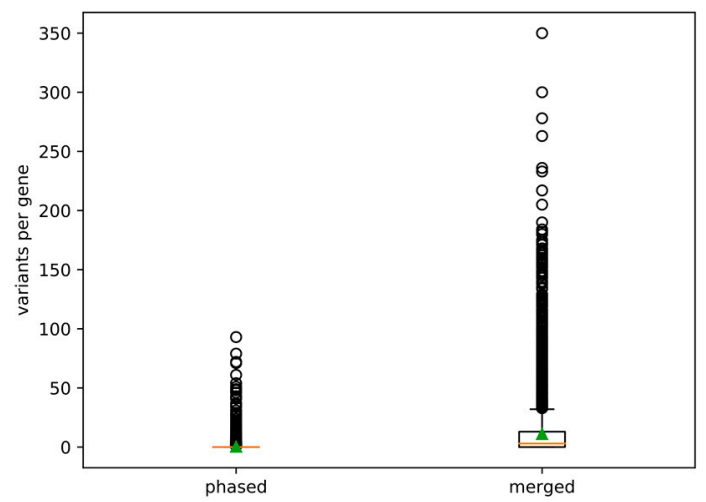

(b)

Figure 1. (a) Distribution of the average sequencing read depth per gene model. Predicted gene models were classified into phase-separated and merged based on the average read depth value deduced from the analysis presented here. The haploid read depth with Illumina short reads ranges from 50 -fold to 150 -fold. (b) Number of heterozygous sequence variants in phase separated and merged genes. The high proportion of heterozygous variants in merged gene models is due to the mapping of reads originating from two different alleles to the same region of the assembly.

\section{Discussion}

The release of genome sequences of many model and crop plants has provided new opportunities for gene identification and studies of genome evolution, both ultimately serving the process of plant breeding [45] by allowing discovery of genes responsible for important agronomic traits and the development of molecular markers associated with these traits. Here, we present the first genome sequence for D. dumetorum, an important crop for Central and Western Africa, and the second genome sequence for the genus. Our assembly offers a great opportunity to understand the evolution of yam and to elucidate some biological constraints inherent to yam including a long growth cycle, poor to non-flowering, polyploidy, vegetative propagation, and a heterozygous genetic background [46]. Yam improvement has been challenging due to these factors preventing the genetic study of important traits in yam [47].

Oxford Nanopore Technology sequencing has proven to be a reliable and affordable technology for sequencing genomes thus replacing Illumina technique for de novo genome sequencing due to substantially higher assembly continuity $[42,48]$. Large fractions of the genome sequence were separated into phases, while regions with lower heterozygosity are merged into one representative sequence. Coverage analysis with Illumina read mapping allowed to classify predicted gene models as 'phase-separated' or 'merged' based on an average coverage around 100-fold or around 200-fold, respectively. While this distinction is possible at the gene model level, whole contigs cannot be classified this way. Several Mbp long contigs comprise alternating phase-separated and merged regions. Therefore, it is likely that the contigs represent a mixture of both haplophases with the risk of switching between phases at each merged region. Since the haplophases cannot be resolved continuously through low heterozygosity regions, purging of contigs to reduce the assembly into a representation of the haploid genome might be advantageous for some applications in the future. The bimodal coverage distribution (Figure 1a) supports the assumption that D. dumetorum Ibo sweet 3 has a diploid genome. This is supported by Smudgeplot for three out of four k-mer sizes tested while 
the shortest k-mer size used (19) finds indications for tetraploidy. Since a high ploidy would result in more distinct coverage peaks as observed for a genome with up to pentaploid parts [42], we assume that the genome is diploid. The weak hint for tetraploidy might be due to a whole genome duplication event early in the diversification of the genus. The $\mathrm{N}_{50}$ of $3.2 \mathrm{Mbp}$ is in the expected range for a long read assembly of a highly heterozygous plant species which contains some quite repetitive sequences as others reported similar values before [49]. Due to regions of merged haplophases the total assembly size of $485 \mathrm{Mbp}$ is smaller than expected for a fully phase-separated "diploid" genome sequence based on the haploid genome size estimation of $322 \mathrm{Mbp}$.

We noticed an increase in the number of BUSCOs through several polishing rounds. Initial assemblies of long reads can contain numerous short insertions and deletions as these are the major error type in ONT reads [50]. As a result, the identification of CDSs and deduced open reading frames is hindered through apparent disruptions of some CDS. Through the applied polishing steps, the number of such apparent frame shifts is reduced thus leading to an increase of detected BUSCOs.

D. dumetorum has 36 chromosomes [12], so with 924 contigs we are far from chromosome-level resolution but considerably better than the other genome assembly published in the genus, that of $D$. rotundata with 40 chromosomes [8]. Knuth [51] circumscribed D. dumetorum and D. rotundata in two distant sections D. sect. Lasiophyton and D. sect. Enantiophlyllum, respectively. Additionally, phylogenetically the two species are quite distantly related with a last common ancestor about 30 million years ago [11,52]. Comparing our predicted peptides to the D. rotundata peptide set [8], we identified about 9800 single copy orthologs (6475 in the whole set of 35,269 gene models plus 3352 with a relation of one gene in D. rotundata and two phase-separated alleles in D. dumetorum) which could elucidate the evolutionary history of those species. The total number of predicted protein-encoding gene models was determined to be 35,269, but this number includes two copies of about 11,300 gene models (see Figure 1) as these are represented by two alleles each. The CDS-based pairing we performed detected about 8500 of the theoretical maximum of 11,300 cases which is a good success rate given the fact that close paralogs and also hemizygous genome regions contribute to the detected number of phase-separated gene models. If phase-separated gene models (alleles) are excluded, a number of about 24,000 genes would result for $D$. dumetorum. This fits to the range detected in other plant genomes [53,54]. The BUSCO results support this interpretation with about $40 \%$ of BUSCOs that occur with exactly two copies. Therefore, the true number of protein-encoding genes of a haploid $D$. dumetorum (Trifoliate yam) genome could be around 25,000, also considering that the BUSCO analysis indicated by $5.8 \%$ missing BUSCOs that still a small fraction of the genome is not represented in the current assembly. This gene number fits well to gene numbers of higher plants based on all available annotations at NCBI/EBI [54]. The average length of genes and the number of encoded amino acids are in the same range as previously observed for other plant species from diverse taxonomic groups $[33,55]$.

It should be noted that the assignment of $D$. dumetorum sequences to the $D$. rotundata pseudochromosomes and indirectly the respective linkage groups contain the risk of incorrect assignments. However, although $D$. rotundata and D. dumetorum are evolutionary separated, D. rotundata is the most closely related species with genetic and genomic resources.

Our draft genome has the potential to provide the basis for new ways to breed with D. dumetorum, for example avoiding the post-harvest hardening phenomenon, which begins within $24 \mathrm{~h}$ after harvest and makes it necessary to process the tubers within this time to allow consumption [2]. The family Dioscoreaceae consists of more than 800 species [56] and the post-harvest hardening phenomenon has only been reported from $D$. dumetorum [57], outlining the singularity of this species among yam species. We predicted a large number of genes, which will include putative genes controlling the post-harvest hardening on D. dumetorum and many useful bioactive compounds detected in this yam species, which is considered the most nutritious and valuable from a phytomedical point of view [58]. Ongoing work will try to identify these genes and polymorphisms for making them available for subsequent breeding.

In summary, we present the first de novo nuclear genome sequence assembly of $D$. dumetorum with very good contiguity and partially separated phases. Our assembly has no ambiguous bases and 
offers a well applicable gene model annotation. This assembly unraveled the genomic structure of $D$. dumetorum to a large extent and will serve as a reference genome sequence for yam breeding by helping to identify and develop molecular markers associated with relevant agronomic traits, and to understand the evolutionary history of $D$. dumetorum and yam species in general.

Supplementary Materials: The following are available online at http://www.mdpi.com/2073-4425/11/3/274/s1, File S1: Sequencing overview with ENA identifiers of runs. File S2: Results of BUSCO analysis of different assembly versions. File S3: AGP file describing contig assignment to D. rotundata pseudochromosomes. File S4: Genome size estimation overview using four different tools. File S5: General statistics of different assembly versions. File S6: Comparison of results from different gene prediction approaches. File S7: Orthogroups of predicted peptides of D. rotundata and D. dumetorum. File S8: Similarity of D. dumetorum and D. rotundata based on single copy orthologs. File S9: Functional annotation of predicted genes in the D. dumetorum genome sequence. File S10: Consensus sequences of repeat elements detected in the D. dumetorum genome sequence. File S11: Average short read mapping coverage of predicted genes in the D. dumetorum genome sequence. File S12: Results from Smudgeplot analyses.

Author Contributions: C.S., B.P., D.C.A., and B.W. designed the study. C.S. collected the sample. B.P. performed DNA extraction, ONT sequencing, and genome assembly. P.V. performed Illumina sequencing. C.S. and B.P. processed the assembly. B.P. performed gene prediction and evaluation. C.S. and B.P. wrote the initial draft. B.W. and D.C.A. revised the manuscript. All authors read and approved the final version of the manuscript.

Funding: This research was partly funded by the German Academic Exchange Service (DAAD, No. 57299294) with a fellowship to CS.

Acknowledgments: We thank the Appropriate Development for Africa foundation (ADAF) for the yam collection in Cameroon and for permission to study the plants in Germany in the framework of our mutual protocol agreement. We also thank the German Society of Botany (DBG) for supporting the research stay of CS in Bielefeld. We acknowledge support for the Article Processing Charge by the Deutsche Forschungsgemeinschaft and the Open Access Publication Fund of Bielefeld University.

Conflicts of Interest: The authors declare no conflict of interest.

\section{References}

1. Viruel, J.; Forest, F.; Paun, O.; Chase, M.W.; Devey, D.; Couto, R.S.; Segarra-Moragues, J.G.; Catalan, P.; Wilkin, P. A nuclear Xdh phylogenetic analysis of yams (Dioscorea Dioscoreaceae) congruent with plastid trees reveals a new Neotropical lineage. Bot. J. Linn. Soc. 2018, 187, 232-246. [CrossRef]

2. Sefa-Dedeh, S.; Afoakwa, E.O. Biochemical and textural changes in trifoliate yam Dioscorea dumetorum tubers after harvest. Food Chem. 2002, 79, 27-40. [CrossRef]

3. Alozie, Y.E.; Akpanabiatu, M.; Eyong, E.U.; Umoh, I.B.; Alozie, G. Amino Acid Composition of Dioscorea dumetorum Varities. Pak. J. Nutr. 2009, 8, 103-105.

4. Ferede, R.; Maziya-Dixon, B.; Alamu, O.E.; Asiedu, R. Identification and quantification of major carotenoids of deep yellow-fleshed yam (tropical Dioscorea dumetorum). J. Food Agric. Environ. 2010, 8, 160-166.

5. Nimenibo-Uadia, R.; Oriakhi, A. Proximate, Mineral and Phytochemical Composition of Dioscorea dumetorium Pax. J. Appl. Sci. Environ. Manag. 2017, 21, 771-774.

6. Iwu, M.M.; Okunji, C.O.; Ohiaeri, G.O.; Akah, P.; Corley, D.; Tempesta, M.S. Hypoglycaemic activity of dioscoretine from tubers of Dioscorea dumetorum in normal and alloxan diabetic rabbits. Plant. Med. 1990, 56, 264-267. [CrossRef]

7. Siadjeu, C.; Panyoo, E.A.; Toukam, G.M.S.; Bell, J.M.; Nono, B.; Medoua, G.N. Influence of Cultivar on the Postharvest Hardening of Trifoliate Yam (Dioscorea dumetorum) Tubers. Adv. Agric. 2016, 2658983. [CrossRef]

8. Tamiru, M.; Natsume, S.; Takagi,H.; White, B.; Yaegashi, H.; Shimizu, M.; Yoshida, K.; Uemura, A.; Oikawa, K.; Abe, A.; et al. Genome sequencing of the staple food crop white Guinea yam enables the development of a molecular marker for sex determination. BMC Biol. 2017, 15, 86. [CrossRef]

9. Cormier, F.; Lawac, F.; Maledon, E.; Gravillon, M.C.; Nudol, E.; Mournet, P.; Vignes, H.; Chaïr, H.; Arnau, G. A reference high-density genetic map of greater yam (Dioscorea alata L.). Theor. Appl. Genet. 2019, 132, 1733-1744. [CrossRef]

10. Ngo Ngwe, M.F.; Omokolo, D.N.; Joly, S. Evolution and Phylogenetic Diversity of Yam Species (Dioscorea spp.): Implication for Conservation and Agricultural Practices. PLoS ONE 2015, 10, e0145364. [CrossRef] 
11. Magwe-Tindo, J.; Wieringa, J.J.; Sonke, B.; Zapfack, L.; Vigouroux, Y.; Couvreur, T.L.P.; Scarcelli, N. Complete plastome sequences of 14 African yam species (Dioscorea spp.). Mitochondrial DNA Part B-Resour. 2019, 4, 74-76. [CrossRef]

12. Miege, J. Nombres chromosomiques et répartition géographique de quelques plantes tropicales et équatoriales. Revue de Cytologie et de Biologie Végétales 1954, 15, 312-348.

13. Hui-Chen, C.; Mei-Chen, C.; Ping-Ping, L.; Chih-Tsun, T.; Fang-Ping, D. A cytotaxonomic study on Chinese Dioscorea, L.-The chromosome numbers and their relation to the origin and evolution of the genus. J. Syst. Evol. 1985, 23, 11-18.

14. Siadjeu, C.; Mayland-Quellhorst, E.; Albach, D.C. Genetic diversity and population structure of trifoliate yam (Dioscorea dumetorum Kunth) in Cameroon revealed by genotyping-by-sequencing (GBS). BMC Plant Biol. 2018, 18, 359. [CrossRef]

15. Rosso, M.G.; Li, Y.; Strizhov, N.; Reiss, B.; Dekker, K.; Weisshaar, B. An Arabidopsis thaliana T-DNA mutagenised population (GABI-Kat) for flanking sequence tag based reverse genetics. Plant Mol. Biol. 2003, 53, 247-259. [CrossRef]

16. Vurture, G.W.; Sedlazeck, F.J.; Nattestad, M.; Underwood, C.J.; Fang, H.; Gurtowski, J.; Schatz, M.C. GenomeScope: Fast reference-free genome profiling from short reads. Bioinformatics 2017, 33, 2202-2204. [CrossRef]

17. Sun, H.; Ding, J.; Piednoel, M.; Schneeberger, K. findGSE: Estimating genome size variation within human and Arabidopsis using k-mer frequencies. Bioinformatics 2018, 34, 550-557. [CrossRef]

18. Liu, B.; Shi, Y.; Yuan, J.; Hu, X.; Zhang, H.; Li, N.; Li, Z.; Chen, Y.; Mu, D.; Fan, W. Estimation of genomic characteristics by analyzing k-mer frequency in de novo genome projects. arXiv 2013, arXiv:1308.2012.

19. Marcais, G.; Kingsford, C. A fast, lock-free approach for efficient parallel counting of occurrences of k-mers. Bioinformatics 2011, 27, 764-770. [CrossRef]

20. Pucker, B. Mapping-based genome size estimation. bioRxiv 2019. [CrossRef]

21. Ranallo-Benavidez, T.R.; Jaron, K.S.; Schatz, M.C. GenomeScope 2.0 and Smudgeplots: Reference-free profiling of polyploid genomes. bioRxiv 2019, 747568. [CrossRef] [PubMed]

22. Koren, S.; Walenz, B.P.; Berlin, K.; Miller, J.R.; Bergman, N.H.; Phillippy, A.M. Canu: Scalable and accurate long-read assembly via adaptive k-mer weighting and repeat separation. Genome Res. 2017, 27, 722-736. [CrossRef] [PubMed]

23. Li, H. Minimap2: Pairwise alignment for nucleotide sequences. Bioinformatics 2018, 34, 3094-3100. [CrossRef]

24. Vaser, R.; Sović, I.; Nagarajan, N.; Šikić, M. Fast and accurate de novo genome assembly from long uncorrected reads. Genome Res. 2017, 27, 737-746. [CrossRef] [PubMed]

25. Li, H. Aligning sequence reads, clone sequences and assembly contigs with BWA-MEM. arXiv 2013, arXiv:1303.3997.

26. Walker, B.J.; Abeel, T.; Shea, T.; Priest, M.; Abouelliel, A.; Sakthikumar, S.; Cuomo, C.A.; Zeng, Q.; Wortman, J.; Young, S.K.; et al. Pilon: An integrated tool for comprehensive microbial variant detection and genome assembly improvement. PLoS ONE 2014, 9, e112963. [CrossRef]

27. Pucker, B.; Holtgräwe, D.; Rosleff Sörensen, T.; Stracke, R.; Viehöver, P.; Weisshaar, B. A De Novo Genome Sequence Assembly of the Arabidopsis thaliana Accession Niederzenz-1 Displays Presence/Absence Variation and Strong Synteny. PLoS ONE 2016, 11, e0164321. [CrossRef]

28. Tang, H.; Zhang, X.; Miao, C.; Zhang, J.; Ming, R.; Schnable, J.C.; Schnable, P.S.; Lyons, E.; Lu, J. ALLMAPS: Robust scaffold ordering based on multiple maps. Genome Biol. 2015, 16, 3. [CrossRef]

29. Pucker, B.; Holtgräwe, D.; Weisshaar, B. Consideration of non-canonical splice sites improves gene prediction on the Arabidopsis thaliana Niederzenz-1 genome sequence. BMC Res. Notes 2017, 10, 667. [CrossRef]

30. Simão, F.A.; Waterhouse, R.M.; Ioannidis, P.; Kriventseva, E.V.; Zdobnov, E.M. BUSCO: Assessing genome assembly and annotation completeness with single-copy orthologs. Bioinformatics 2015, 31, 3210-3212. [CrossRef]

31. Keller, O.; Kollmar, M.; Stanke, M.; Waack, S. A novel hybrid gene prediction method employing protein multiple sequence alignments. Bioinformatics 2011, 27, 757-763. [CrossRef] [PubMed]

32. Smit, A.F.A.; Hubley, R.; Green, P. RepeatMasker Open-4.0. Available online: http://www.repeatmasker.org (accessed on 2 December 2018).

33. Pucker, B.; Feng, T.; Brockhington, S. Next generation sequencing to investigate genomic diversity in Caryophyllales. bioRxiv 2019. [CrossRef]

34. Altschul, S.F.; Gish, W.; Miller, W.; Myers, E.W.; Lipman, D.J. Basic local alignment search tool. J. Mol. Biol. 1990, 215, 403-410. [CrossRef] 
35. Cheng, C.Y.; Krishnakumar, V.; Chan, A.; Thibaud-Nissen, F.; Schobel, S.; Town, C.D. Araport11: A complete reannotation of the Arabidopsis thaliana reference genome. Plant J. 2017, 89, 789-804. [CrossRef] [PubMed]

36. Jones, P.; Binns, D.; Chang, H.Y.; Fraser, M.; Li, W.; McAnulla, C.; McWilliam, H.; Maslen, J.; Mitchell, A.; Nuka, G.; et al. InterProScan 5: Genome-scale protein function classification. Bioinformatics 2014, 30, 1236-1240. [CrossRef] [PubMed]

37. Lowe, T.M.; Eddy, S.R. tRNAscan-SE: A program for improved detection of transfer RNA genes in genomic sequence. Nucleic Acids Res. 1997, 25, 955-964. [CrossRef] [PubMed]

38. Chan, P.P.; Lowe, T.M. tRNAscan-SE: Searching for tRNA Genes in Genomic Sequences. In Gene Prediction: Methods and Protocols; Kollmar, M., Ed.; Springer: Berlin/Heidelberg, Germany, 2019; Volume 1962, pp. 1-14.

39. Nawrocki, E.P.; Eddy, S.R. Infernal 1.1: 100-fold faster RNA homology searches. Bioinformatics 2013, 29, 2933-2935. [CrossRef]

40. Kalvari, I.; Argasinska, J.; Quinones-Olvera, N.; Nawrocki, E.P.; Rivas, E.; Eddy, S.R.; Bateman, A.; Finn, R.D.; Petrov, A.I. Rfam 13.0: Shifting to a genome-centric resource for non-coding RNA families. Nucleic Acids Res. 2018, 46, D335-D342. [CrossRef]

41. Flynn, J.M.; Hubley, R.; Goubert, C.; Rosen, J.; Clark, A.G.; Feschotte, C.; Smit, A.F. RepeatModeler2: Automated genomic discovery of transposable element families. bioRxiv 2019. [CrossRef]

42. Pucker, B.; Ruckert, C.; Stracke, R.; Viehover, P.; Kalinowski, J.; Weisshaar, B. Twenty-Five Years of Propagation in Suspension Cell Culture Results in Substantial Alterations of the Arabidopsis Thaliana Genome. Genes 2019, 10, 671. [CrossRef]

43. Baasner, J.S.; Howard, D.; Pucker, B. Influence of neighboring small sequence variants on functional impact prediction. bioRxiv 2019. [CrossRef]

44. Emms, D.M.; Kelly, S. OrthoFinder: Phylogenetic orthology inference for comparative genomics. Genome Biol. 2019, 20, 238. [CrossRef] [PubMed]

45. Ruggieri, V.; Alexiou, K.G.; Morata, J.; Argyris, J.; Pujol, M.; Yano, R.; Nonaka, S.; Ezura, H.; Latrasse, D.; Boualem, A.; et al. An improved assembly and annotation of the melon (Cucumis melo L.) reference genome. Sci. Rep. 2018, 8, 8088. [CrossRef] [PubMed]

46. Mignouna, H.D.; Abang, M.M.; Asiedu, R. Harnessing modern biotechnology for tropical tuber crop improvement: Yam (Dioscorea spp.) molecular breeding. Afr. J. Biotechnol. 2003, 2, 12.

47. Mignouna, H.D.; Abang, M.M.; Asiedu, R. Genomics of Yams, a Common Source of Food and Medicine in the Tropics. In Genomics of Tropical Crop Plants; Moore, P.H., Ming, R., Eds.; Springer: New York, NY, USA, 2008; pp. 549-570. [CrossRef]

48. Michael, T.P.; Jupe, F.; Bemm, F.; Motley, S.T.; Sandoval, J.P.; Lanz, C.; Loudet, O.; Weigel, D.; Ecker, J.R. High contiguity Arabidopsis thaliana genome assembly with a single nanopore flow cell. Nat. Commun. 2018, 9, 541. [CrossRef]

49. Paajanen, P.; Kettleborough, G.; Lopez-Girona, E.; Giolai, M.; Heavens, D.; Baker, D.; Lister, A.; Cugliandolo, F.; Wilde, G.; Hein, I.; et al. A critical comparison of technologies for a plant genome sequencing project. Gigascience 2019, 8, giy163. [CrossRef]

50. Salmela, L.; Walve, R.; Rivals, E.; Ukkonen, E. Accurate self-correction of errors in long reads using de Bruijn graphs. Bioinformatics 2017, 33, 799-806. [CrossRef]

51. Knuth, R. Dioscoreaceae. In Das Pflanzenreich; Engelr, A., Ed.; Engelmann, W.: Leipzig, Germany, 1924.

52. Viruel, J.; Segarra-Moragues, J.G.; Raz, L.; Forest, F.; Wilkin, P.; Sanmartin, I.; Catalan, P. Late Cretaceous-Early Eocene origin of yams (Dioscorea, Dioscoreaceae) in the Laurasian Palaearctic and their subsequent Oligocene-Miocene diversification. J. Biogeogr. 2016, 43, 750-762. [CrossRef]

53. Wendel, J.F.; Jackson, S.A.; Meyers, B.C.; Wing, R.A. Evolution of plant genome architecture. Genome Biol. 2016, 17, 37. [CrossRef]

54. Pucker, B.; Brockington, S.F. Genome-wide analyses supported by RNA-Seq reveal non-canonical splice sites in plant genomes. BMC Genom. 2018, 19, 980. [CrossRef]

55. Pucker, B.; Holtgräwe, D.; Stadermann, K.B.; Frey, K.; Huettel, B.; Reinhardt, R.; Weisshaar, B. A chromosome-level sequence assembly reveals the structure of the Arabidopsis thaliana Nd-1 genome and its gene set. PLoS ONE 2019, 14, e0216233. [CrossRef] [PubMed]

56. Barton, H. Yams: Origins and Development. Encyclopaedia Glob. Archaeol. 2014, 7943-7947. 
57. Treche, S.; Delpeuch, F. Physiologie Vegetale-Mise en evidence de l'apparition d'un epaississement membranaire dans le parenchyme des tubercules de Dioscorea dumetorum au cours de la conservation. Comptes Rendus de l'Académie des Sciences. Série D: Sciences Naturelles 1979, 288, 67-70.

58. Price, E.J.; Wilkin, P.; Sarasan, V.; Fraser, P.D. Metabolite profiling of Dioscorea (yam) species reveals underutilised biodiversity and renewable sources for high-value compounds. Sci. Rep. 2016, 6, 29136. [CrossRef]

(C) 2020 by the authors. Licensee MDPI, Basel, Switzerland. This article is an open access article distributed under the terms and conditions of the Creative Commons Attribution (CC BY) license (http://creativecommons.org/licenses/by/4.0/). 